\title{
Contributions of general practitioners in China facing the COVID-19 outbreak
}

Kai Chen, Cuifei Liang, Ruihong Liu

AT THE END OF DECEMBER 2019, some 'unexplained pneumonia' cases were reported in Wuhan, China. Around this time, similar cases also appeared all over the world. Subsequent virus isolation confirmed that the pneumonia was caused by SARS-CoV-2 infection, and the disease was officially named COVID-19. ${ }^{1}$

The COVID-19 outbreak was a test for China's basic level public health system and brought severe challenges to medical institutions. ${ }^{2}$ At present, the domestic epidemic in China has been effectively controlled, and life and production have started to return to normal. During the pandemic, general practitioners (GPs) have played the crucial part of health gatekeepers, which should be acknowledged and appreciated. ${ }^{2}$

\section{Contributions of general practitioners to COVID-19 prevention}

\section{Detecting cases and curbing the outbreak}

GPs act as both gatekeepers and health facilitators by enabling the community to build a barrier against COVID-19. GPs spend a substantial amount of time working in the community and are familiar with the state of the community's residents, including their overall health, living habits and human mobility. GPs work together with local community workers in a 'joint defence team' led by the neighbourhood committee, just as they did conventionally before the outbreak, while community workers provide nonmedical support to residents. ${ }^{3}$ During the COVID-19 outbreak, GPs are seen everywhere, from community health centres to fever clinics in hospitals, fulfilling their duties in detecting cases. GPs also establish temperature monitoring stations at the entrance of each district to help verify personal information and carry out health checks for residents. Medical isolation and observation are indicated if there are any signs of illness, contact with confirmed cases or travel to high-risk areas. The suspected cases will be immediately referred to a fever clinic at a designated hospital by a dedicated staff member for detailed epidemiological investigation and nucleic acid detection.

\section{Providing continuity of care}

According to the policy requirements for epidemic prevention in China, most residents in high-risk areas are required to live in isolation consciously. ${ }^{4}$ But for patients with chronic diseases and elderly individuals who live alone, continuous and comprehensive medical services are still needed. To solve the inconvenience of patients going to hospital and purchasing medicines, GPs communicate with the patients via telephone and online platforms and deliver medication to patients in various communities. Despite the challenges presented by COVID-19, GPs remain committed to assisting their patients to take medication regularly to maintain continuity of treatment. They also observe the treatment effect, monitor patients for adverse drug reactions and dynamically adjust the treatment plan to obtain a good prognosis. ${ }^{3}$

\section{Providing psychological crisis intervention}

Facing an epidemic and closed management, people's fears and anxiety spread faster than the virus; these psychological consequences can be as devastating as physical health problems. To reduce the mental health effects and avoid further social panic, GPs provide guidance and counselling in the form of face-to-face interviews, communication hotlines and online consultation services. They also make the preliminary evaluation, diagnosis and treatment plans for residents with psychological conditions and cooperate with psychological experts to carry out treatment. ${ }^{3}$

\section{Carrying out community health education}

GPs are the best executives of community health education and behaviour intervention during and prior to the pandemic. Despite the challenges presented by COVID-19, GPs remain 
committed to assisting their patients with advice and support to wear masks properly, wash hands frequently and keep a safe distance. WeChat official accounts, leaflets and bulletin boards are the common publicity channels delivered by large groups of GPs. These educational materials are usually created by GPs and the National Health Commission in a unified manner to avoid confusing the community. Furthermore, GPs widely publicise information regarding disease protection and healthy lifestyles to reduce the gathering of people and minimise the risk of epidemic transmission. ${ }^{3}$

\section{First published 29 October 2021.}

\section{Authors}

Kai Chen MMed, Resident, Department of Family Medicine, University of Hong Kong-Shenzhen

Hospital, Shenzhen, China

Cuifei Liang BMED, Resident, Department of Family Medicine, University of Hong Kong-Shenzhen

Hospital, Shenzhen, China

Ruihong Liu MD, Associate Consultant, Department of Family Medicine, University of Hong Kong-Shenzhen Hospital, Shenzhen, China; Assistant Professor,

Department of Family Medicine and Primary Care, The University of Hong Kong, Hong Kong, China

Competing interests: None.

Funding: None.

Provenance and peer review: Not commissioned, externally peer reviewed.

Citation: Chen K, Liang C, Liu R. Contributions of general practitioners in China facing the COVID-19 outbreak. Aust J Gen Pract 2021;50 Suppl 51. doi: 10.31128/AJGP-COVID-51.

Correspondence to:

liurh@hku-szh.org

\section{References}

1. Sharma A, Ahmad Farouk I, Lal SK. COVID-19: A review on the novel coronavirus disease evolution, transmission, detection, control and prevention. Viruses 2021;13(2):202. doi: 10.3390/ v13020202.

2. $\mathrm{Wu} Z$, McGoogan JM. Characteristics of and important lessons from the coronavirus disease 2019 (COVID-19) outbreak in China: Summary of a report of 72314 cases from the Chinese Center for Disease Control and Prevention. JAMA 2020;323(13):1239-42. doi: 10.1001/ jama.2020.2648.

4. Li DKT, Zhu S. Contributions and challenges of general practitioners in China fighting against the novel coronavirus crisis. Fam Med Community Health 2020;8(2):e000361. doi: 10.1136/fmch2020-000361.

5. Umakanthan S, Sahu P, Ranade AV, et al. Origin, transmission, diagnosis and management of coronavirus disease 2019 (COVID-19). Postgrad Med J 2020;96(1142):753-58. doi: 10.1136/ postgradmedj-2020-138234. 